\title{
Care for Both Partners before Conception: The Logical Starting Place To Improve Fertility and Every Aspect of Reproduction
}

\author{
Janette E Roberts* \\ Diploma Clinical Nutrition, USA \\ *Corresponding author: Janette E Roberts, Diploma Clinical Nutrition, USA.
}

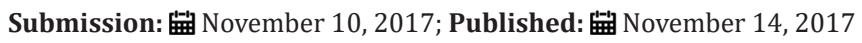

\section{Opinion}

With so much attention and media exposure devoted to the emotive and ever-increasing issue of infertility, the bigger picture of generally-compromised reproductive outcomes is often overlooked. It's now thirty five years since I began promoting preconception healthcare for both prospective parents as the surest way to conceiving and birthing the healthiest baby possible. But through all my years of promoting the benefits of preconception healthcare, I've been aware that most individuals wish they'd known what a huge opportunity (and responsibility of course) actually rests with them! My books, webinars, presentations and other educational content are for anybody who might ever become a Mum or a Dad - the physical, mental and emotional health of their children is in their hands and has its foundation in their own health before conception.

The idea of providing optimal conditions for pregnancy, well before the mother-to-be is even pregnant, is rapidly gaining popularity all over the world. Many experts see the four months immediately preceding conception as a critical stage in the reproductive process - the true 'first trimester' if you will! Factors such as nutritional, lifestyle, environmental, and emotional issues are taken into consideration, with both partners equally involved in the preparations to provide their future child with physical and mental health advantages that will last that child's entire lifetime.

If you're in any doubt, the research is clear. For a family to enjoy a lifetime of better health, their journey must start before conception. My own work in the area of preconception health was inspired by the work of Foresight. Established in the UK in 1978, The Association for the Promotion of Preconceptual Care has involved researchers \& research institutions from around the globe. The studies have covered a variety of areas identifying the adverse effects on reproduction of a cocktail of factors that include nutritional deficits, exogenous hormones, heavy metals, pesticides, smoking, alcohol, electromagnetic radiation, stress and more.

In April 2006, United States' CDC (Centers for Disease Control and Prevention) National Centre on Birth Defects and Developmental
Disabilities released health guidelines for all women who could fall pregnant, even those who had no plans to become a parent. Associate Director for the development of the program stated: 'If you want to prevent any damage to the baby while the fetal organs are being formed, you need to take action before that time. We want couples to consciously think about being ready for pregnancy before pregnancy happens. Improving preconception health will require changes in the knowledge and attitudes and behaviours of persons, families, communities, and institutions (e.g., government and health-care settings). The purpose of preconception care is to improve the health of each woman before any pregnancy and thereby affect the future health of the woman, her child, and her family.'

Professor David Barker physician, epidemiologist and originator of the Barker Hypothesis stated that 'Preventing heart disease \& type 2 diabetes needs to begin before a baby is even conceived.' Barker said research had found low birth-weight babies were at increased risk of heart attacks, strokes, diabetes, high blood pressure and osteoporosis. He said slow foetal growth was primarily determined by a mother's diet - both before and after conception, but particularly before pregnancy. 'Even if mothers eat very little, which happened during the war-time famines in Holland, the baby's still able to grow because if the mother entered pregnancy well-nourished she's got a lot of reserves of protein and fat? Every farmer knows that you don't mate ewes until they're in good condition. It's just an integral part of farming. How come we think people are different?' [1].

But we clearly do think people are different, with current statistics confirming that the human population is ignoring what farmers, stockbreeders and veterinarians understand and practice.
a) 1 couple in 6 is infertile
b) 1 woman in $4(1$ in 3 ?) will miscarry
c) 1 baby in 10 is born prematurely
d) 1 baby in 30 is born with a congenital abnormality 
e) 1 woman in 3 has a Caesarean section (in some regions such as China, and Brazil the number is dramatically higher)

f) 1 woman in 6 suffers from some degree of post-natal depression

g) Only 1 woman in 10 breastfeeds beyond her child's first birthday

h) MIND (metabolic, immune, neurological and digestive) disorders in children are at epidemic levels and rising ... the list goes on.

But those statistics represent a far from auspicious start for the next generation and their families, with the physical, mental and emotional fallout from these statistics apparent from before conception, with chronic ill-health, accelerated ageing, degenerative disease and disability and reduced life expectancy the end results. But the studies carried out by Foresight, in conjunction with Surrey University UK [2], show that most reproductive problems can be avoided if both prospective parents are in excellent health before they conceive a child. The results of Foresight's several studies are compelling. Let's look at the first one, which was published in the Journal of Natural and Environmental Medicine, 1995: When 367 couples followed the full preconception program there was not one miscarriage in the entire study group.

a) There were no peri-natal deaths and no malformations. (Without preconception health care, there would be about 70 miscarriages and 12 malformations in the same group).

b) $37 \%$ of the couples had experienced previous fertility problems (from 1 to 10 years duration). By the end of the study, which ran for 18 months, $81 \%$ of those couples had a live healthy baby.

c) No baby was born before 36 weeks.

d) No baby was lighter than 5lb. 3oz (2368g)

e) No baby was admitted to Special Care

The absence of premature and very low birth weight babies when couples practice preconception healthcare is another significant factor in light of Professor David Barker's findings [3].

So what's involved in preconception healthcare for both partners? Top of the list are self-help, health-promotion measures that are the responsibility of the individual. Then come the treatments and support that can be delivered by Practitioners of Natural Medicine and/or Integrative Medical Doctors and finally the products-adaptogens, nutritional support, probiotics, purified water, low-toxicity skin care products and much more, that best support the combined efforts of the individual and his/her healthcare providers $[4,5]$.

So knowing the huge benefits that can be achieved with blanket preconception recommendations that are faithfully followed by both prospective parents, where does my current focus lie? Unsurprisingly, it's not in doing more research to prove what we already know. However, one potential new area of research would be to identify genetic predispositions for a particular condition and how the switch for that gene can be turned off by dietary and lifestyle modifications. Second area of huge importance is in making this information readily do-able and as widely available as possible.

Let's consider the delivery of that information. With multiple media through which to spread the word and clever ways to engage and motivate audiences, we can now make this information heard by tens of millions not just tens of thousands. The goal of my company, Health HQ Global is to reach prospective parents before they see IVF as their only option or before they are destined to spend a lifetime caring for a child with a physical or mental health issue that could have been prevented. But our recommendations don't stop there- the same program that delivers such awesome reproductive results will ensure a lifetime of better health for the whole family - in particular vital and energetic senior years free of chronic degenerative disease!

\section{References}

1. (2005) Chronic disease may start pre-conception. Sydney Morning Herald.

2. Naish F, Roberts J (1996) The Natural Way to Better BabiesPreconception Healthcare for Prospective Parents. Random House, USA.

3. Ward N, Keith Eaton (1995) Preconceptional Care and Pregnancy Outcome. Journal of Nutritional and Environmental Medicine 5(2): 205208.

4. Trace Elements and Preconceptual Care. Association for the Promotion of Preconceptual Care UK.

5. Health Professionals' Guide to Preconception Care. Association for the Promotion of Preconceptual Care UK. 